\title{
Evaluation of the Additives' Behaviour to Determine the Best Modifier for Improving Asphalt Performance at High Temperature
}

\author{
Ismail Bakheit Eldouma (i) and Huang Xiaoming \\ School of Transportation Engineering, Southeast University, Nanjing 211189, China \\ Correspondence should be addressed to Huang Xiaoming; huangxm@seu.edu.cn
}

Received 20 June 2020; Revised 30 November 2020; Accepted 28 December 2020; Published 8 January 2021

Academic Editor: Yuqing Zhang

Copyright ( 2021 Ismail Bakheit Eldouma and Huang Xiaoming. This is an open access article distributed under the Creative Commons Attribution License, which permits unrestricted use, distribution, and reproduction in any medium, provided the original work is properly cited.

\begin{abstract}
The current study aims to examine the physical and rheological properties at the high-temperature range of $52,58,64,70,76$, and $82^{\circ} \mathrm{C}$, utilizing adjusted asphalt binders. Three kinds of asphalt modifiers were selected such as styrene-butadiene-styrene, ethylene vinyl acetate, and end of life tires. The selected additives were implemented with different contents ranging from $4 \%$ to $7 \%$ by the weight of neat asphalt. Various test methods have been established, such as ductility, viscosity, microscopic inspection, and dynamic shear rheometer experiments. Results proved that the percentage decrease in ductility was $93 \%$, $91 \%$, and $88 \%$ with regard to the adding of the end of life tires (ELTs), ethylene vinyl acetate (EVA), and styrene-butadiene-styrene (SBS) additives, respectively. Outcomes also confirmed that viscosity was boosted from $0.411 \mathrm{~Pa}$.s to $1.249,1.0986$, and 0.9785 Pa.s after adding $7 \%$ of ELTs, 7\% of EVA, and 7\% of SBS, respectively. The rutting parameter and complex shear modulus increased after modification, indicating the excellent performance of asphalt. The conclusions confirmed that the ELTs have fewer agglomerates and have good compatibility before ageing and excellent compatibility after the ageing process. Thus, the ELTs are deemed as efficient dispersion additive for avoiding separation during the storage and handling of the asphalt binder. Lastly, ELTs were accepted as the best enhancer for their positive influence on physical and rheological characteristics, which means higher quality amended bitumen would give higher resistance to permanent deformation.
\end{abstract}

\section{Introduction}

Asphalt is defined as black, viscous material, mixed with course and fine aggregates and fillers that forms strong surfaces when it becomes hard [1]. The bitumen was been utilized in the flexible pavement due to its premium characteristics and outstanding favour rendering $[2,3]$. Asphalt sensitivity to temperature was studied and outcomes proposed that the asphalt is changing in climatic situations which decreases the goodness of asphalt roads, causing different pavement distresses such as high-temperature rutting and low-temperature cracking [4]. The effect of intensive traffic tonnage was evaluated on the asphalt pavements and concluded that the quality of bitumen pavements considerably minimized [5]. The appropriate bitumen amendment must be performed to develop high- quality secure, authoritative, and climate-friendly resilient pavement materials [6]. The physical features and the hightemperature execution improved by employing the various enhancers $[7,8]$. Through the different bitumen enhancers, the ELTs, SBS, and EVA additives have attracted the researchers' attentiveness $[9,10]$.

The experimental research was performed on the shear impedance properties using tafpack super (TPS) and SBSadjusted bitumen binders as well as mixtures. The creep data were measured over a comprehensive framework of the stable shear stresses and permanent deformation of porous asphalt blends for the fixed compressive stress. The rapprochement among the shear strain rate and shear creep modulus of TPS and SBS-amended binders was conducted. The results appeared a similar trend for two modified binders [9]. Bouraima et al. proposed that SBS modifier was 
a suitable choice when he utilized the high-viscosity additive (HVA), road-science-technology (RST), and styrene-butadiene-styrene (SBS) additives [10].

The empirical experimental tests and simulations employed styrene-butadiene rubber and molecular dynamics, respectively, to verify the transference and molecular motion within the amended bitumen. The result concluded that the volumetric properties of bitumen affected by the SBR evidenced to be insensitive to the presence of the styrene-butadiene rubber (SBR) modifier and the addition of the styrene-butadiene rubber increased the collection of asphaltene molecules. Also, outcomes showed the possibility of using the polymer modifiers to enhancing the efficient mechanistic lineaments of the bitumen without influencing the volumetric features [1].

Styrene-butadiene-styrene behaves like an elastic rubber at ambient temperature, and it can be processed like plastic when heated (thermoplastic elastomer). However, SBS and other thermoplastic elastomers can be managed to be rubbery without being cross-linked, thus making them simple to transform into useful shapes. SBS network also reacts with the asphaltene and resin micelles as proved by $[11,12]$. EVA copolymer together with a vinyl acetate is applied as the polymer enhancer, and it has attracted considerable awareness at the scope of the flexible pavements $[13,14]$.

The comparison consideration was investigated among the mechanical execution of the end of life tires and the styrene-butadiene-styrene. The rapprochement is conducted to judge which of them is stable regarding the susceptibility to damage distress. Conclusions presented that the ELT-modified binders are more stable than the styrene-butadiene-styrene adjusted bitumen in the expression of additive concentration [15]. Also, ELTs binders manifested positive trend regarding ageing susceptibility, thermal cracking resistance, and water susceptibility better than SBS. ELTs were introduced to bitumen binders to enhance the bitumen specifications which concluded to grow hardness, increase in flexibility with the high serving temperature, and progress the rutting strength $[16,17]$. The study introduced on ELTs was proved that the asphalt fatigue life was extended and the asphalt ageing rate decreased accordingly [18]. Further, the utilization of the ELTs as bitumen enhancer decreases the rigidity of the bitumen binder at low service temperatures which are appropriate for asphalt low serving temperature execution $[19,20]$. The EVA for the time being is ubiquitous because of its favourable properties such as cheapness, ease of moulding, glossy finish, lightweight, and scarceness of odour contrasting to indigenous rubber. EVA is fundamentally recognized for precise specifications as adhesion characteristics, economy, elasticity, stiffness, and stresscrack resistance [21] and, also, identified as one of the materials, popularly expansive rubber or foam rubber $[22,23]$.

Experimental exams cannot adequately depict the behaviours of emended binders. The high-quality requirements necessary to establish essential engineering features qualified to precisely prophesy the rendering of asphalt binders as well as mixes. Moreover, there was an increasing necessity to grasp the reasons that modified binders perform their trend. The necessity to grasp reasons of modification trends encouraged discussions which connect the rendering and morphology of the modified binders, for procuring preferable conception of reaction among the ingredients of the modified binders to evolving adhesives of harmonious behaviour. In producing the modified binders during a mixing up operation, the proper combination of the modifier in asphalt remains an essential demand for realizing the ideal performance of modified binders. The term additive's morphology herein refers to how the additive distributed in the pure asphalt during the blending process. The modifier's morphology is so complicated to recognize by completely measuring the physical and rheological characteristics. However, there was a mechanism that permits the modifier in asphalt to be viewed immediately via using a microscope $[14,24,25]$. The asphalt-rich phase manifests blackish, whereas modifier-rich stage shows shining and often greenish-yellow colour while noticed via ocular microscope $[26,27]$. The aim of utilizing the fluorescent microscopy is to determine which additive is better in terms of homogeneity, compatibility, and stability with storage or ageing to avoid separation during storage and handling.

In our previous study, we conducted a survey of CRM, TPS, and PP modifiers with different concentrations such as $2,3,3.5$, and 4 wt. $\%$ at medium- and high-temperature ranges of 58 to $88^{\circ} \mathrm{C}$ with the increment of $6^{\circ} \mathrm{C}$. Numerous experiments were performed, represented in the ductility, rotational viscosity, toughness and tenacity, DSR, and wheel tracking test (WTT). The experimental results were compared with each other, and CRM was selected as the most preferred enhancer because of its excellent performance at medium and high temperatures [28].

Therefore, it has an important significance to understand the mechanisms of asphalt and additive interaction for improving the performance of asphalt binders and mixtures effectively and reducing the occurrence of pavement distress. Generally, researchers applied adhesion tests to evaluate the asphalt and modifier or filler interaction ability. Currently, researchers have reached a consensus that the asphalt and filler or modifier interaction is a complex physical-chemical interaction process [29].

However, the crack resistance and permanent deformations of the modified asphalt mixtures with different compositions and structures could be evaluated by using different test methods, and some methods are not very suitable for evaluation due to differences in test equipment and failure modes [30].

The adhesion bonding between modified asphalt binder and aggregate significantly impacted the bitumen performance, structural stability, damage growth, and durability of asphalt pavement. Adhesion promoters are typically used to improve asphalt-aggregate bonding and minimize moisture-related pavement damage, such as cracking and raveling. Zhuang et al. evaluated the effectiveness of plant ash by-product as adhesion promoter to improve asphalt-aggregate adhesion performance. They confirmed that the plant ash significantly improved asphalt-aggregate adhesion [31]. 


\section{Materials and Methods}

\subsection{Materials: ELTs, SBS, and EVA Were Applied with Percentage Concentrations of 4, 5, 6, and $7 w t . \%$}

2.1.1. Asphalt. Asphalt 60/70 penetration grade has been utilized, and its physical properties are explained via Table 1 .

2.1.2. End of Life Tires. End of life tire sizes utilized in the current study passed the sieve no. 80\# and retained on the sieve no. 90\#. ELTs' physical particulars are clarified in Table 2.

2.1.3. Ethylene Vinyl Acetate. EVA is one of the materials popularly recognized as expansive rubber or foam rubber $[22,23]$. The physical as well as mechanical features of the utilized EVA modifier are explained in Table 3.

2.1.4. Styrene-Butadiene-Styrene. SBS behaves as an elastic rubber at ambient temperature, and it can be processed like plastic when heated. Styrene-butadiene-styrene and other thermoplastic elastomers can be managed to be rubbery without being cross-linked, thus making them simple to transform into beneficial shapes. SBS features are shown in Table 4.

\subsection{Methods: Several Methods Were Used to Prepare Laboratory Samples}

2.2.1. Sample Preparation. All amended specimens in the current research were designed utilizing both the high-shear and mechanical blenders. Before the alteration procedures, the amount of $1.5 \mathrm{~kg}$ of the virgin bitumen was heated to $180^{\circ} \mathrm{C}$, and then viscosity of the neat asphalt at $135^{\circ} \mathrm{C}$ was examined, it was equal to $0.411 \mathrm{~Pa}$.s [32]. The various mass concentrations of ELTs, SBS, and EVA with percentages of 4 , 5,6 , and 7 wt. $\%$ added gradually to the neat bitumen binder. At the same time, the blending procedure at a speed of 1600 $\mathrm{r} / \mathrm{min}$ was continued for 1 hour using the fan blender. Prepared asphalt was transmitted to the high velocity blender in a velocity of $2500 \mathrm{r} / \mathrm{min}$ at the stable temperature of $190 \pm 5^{\circ} \mathrm{C}$ for 2 hours $[15,33]$.

For preparing the specimen for the ductility experiment, the amended bitumen was heated to $163^{\circ} \mathrm{C} \pm 5^{\circ} \mathrm{C}$ with stirring and then teemed into mould assembly and put on a brass sheet. To prohibit material from imputation, surfaces of the plate and the mould were painted using an admixture of equal parts of glycerine as well as dextrin. The specimen with the mould was kept in a water bath at $25^{\circ} \mathrm{C}$ for $30-40$ minutes, and then the specimen was trimmed and kept for 85-95 minutes in the water bath [9].

In viscosity test sample preparation, the amount of 10 gram of modified asphalt was heated to $190^{\circ} \mathrm{C}$ with continued stirring and poured into the container. In contrast, the actual test temperature was $135^{\circ} \mathrm{C}$. For equipping the DSR experiment samples, the asphalt specimen was put among two plates with a diameter of $25 \mathrm{~mm}$ and $1 \mathrm{~mm}$ gap.
The Olympus BX51M microscope was applied to examine the amended asphalt's internal microstructure, whereas, for evaluating the binder's rheological properties at a broad domain of temperature, the dynamic shear rheometer instrument was being employed. The epi-fluorescence samples were prepared. The exposed surface began to flow with ultraviolet light (UV) exposure through a microscopic examination. The sample was sandwiched between two glass slides to prevent the specimen from flowing and air interaction to avoid effects on the modified binder's morphology. The essential proceedings in the method of industrialization modified asphalt guaranty the distribution of modifier in asphalt and the reaction between modifier and asphalt to determine the modified bitumen's morphology.

2.2.2. Testing Procedures. Ductility, viscosity, and microscopic test, as well as rheological experiments were applied following the American Society for Testing and Materials (ASTM) specifications. Alterations of the ELTs-, EVA-, and SBS-modified binder specimens were evaluated. Obtained results were contrasted with each other as well as with the same parameters of the pure asphalt binder regarding defining the exceedingly useful modifier for bitumen.

Viscometer device was utilized to measure the viscosity at high temperature $135^{\circ} \mathrm{C}$ and, also, determine the modified asphalt's flow features and assess the workability parameters [34]. The spindle and rotor were utilized for locating viscosity in Pa. s units. Ductility experiment was performed for gauging the stretchy features of amended bitumen. After the sample preparation, parts of mould were taken away, buckle accurately hooked on the experimental instrument without giving the initial strain. The indicator was checked to read zero. The experiment instrument was run to pull the sample horizontally at a velocity of $50 \mathrm{~mm} / \mathrm{min}$ [9].

DSR test was performed to define the modified bitumen binder's impedance to permanent deformations at medium and high temperatures by gauging rheological characteristics such as $\mathrm{G}^{*} / \sin (\delta)$ and $\delta$, as well as $\mathrm{G}^{*}[35]$. The $\mathrm{G}^{*} / \sin (\delta), \delta$, and $G^{*}$ for amended bitumen were gauged at $52,58,64,70,76$, and $82^{\circ} \mathrm{C}$, and constant loading frequency of $1.6 \mathrm{~Hz}$ by using two plates with the diameter of a $25 \mathrm{~mm}$ and a $1 \mathrm{~mm}$ gap.

The epi-fluorescence microscopy has been deemed a vital device for characterizing the in-situ constitutional and alchemy influence of enhancers within bitumen to determine their impacts on amended binder rendering. Epifluorescence microscopy was used to observe the modifier dispersion degree in asphalt for different dosages of EVA, ELTs, and SBS additives. In this study, epi-fluorescence microscopy is presented to determine the microstructural analysis of the modified binders and to distinguish through the asphalt phase from the modifier dispersion and also to determine the compatibility between the modifier and asphalt phase in order to prevent segregation during the storage and handling; as well as to improve the overall pavement performance in considering the completeness of the modified binders during manufacture to their achievement at the bitumen stratum. 
TABle 1: Physical properties of the base asphalt.

\begin{tabular}{lccc}
\hline Test & Method & Quantity & Specification limits \\
\hline Penetration at $25^{\circ} \mathrm{C}, 100 \mathrm{~g}, 5 \mathrm{~s}(0.1 \mathrm{~mm})$ & ASTM D5 & 64 & $60-70$ \\
Ductility $\left(25^{\circ} \mathrm{C}, 5 \mathrm{~cm} / \mathrm{min}\right)(\mathrm{cm})$ & ASTM D113 & 150 & Min 100 \\
Kinematic viscosity at $135^{\circ} \mathrm{C}(\mathrm{Pa} . \mathrm{s})$ & ASTM D2170 & 0.411 & - \\
Softening point $(\mathrm{R} \& \mathrm{~B}$ method $)\left({ }^{\circ} \mathrm{C}\right)$ & ASTM D36 & 49 & Min 46.0 \\
Flash point $\left({ }^{\circ} \mathrm{C}\right)$ & ASTM D92 & 317 & - \\
Fire point $\left({ }^{\circ} \mathrm{C}\right)$ & ASTM D92 & 331 & - \\
Specific gravity at $25^{\circ} \mathrm{C}\left(\mathrm{g} / \mathrm{cm}^{3}\right)$ & ASTM D70 & 1.043 & - \\
Loss on heat $(\%)$ & ASTM D-6 & 0.04 & - \\
Dynamic viscosity at $60^{\circ} \mathrm{C}(\mathrm{Pa} . \mathrm{s})$ & ASTM D2171 & 0.410 & - \\
\hline
\end{tabular}

TABLE 2: Ingredients and features of ELTs.

\begin{tabular}{lc}
\hline Component & Content (\%) \\
\hline Ash content & 5 \\
Acetone extract & 11 \\
Natural rubber content & 25.8 \\
Rubber hydrocarbon content & 37 \\
Carbon black content & 21.2 \\
Relative density & 1.2 \\
\hline
\end{tabular}

TABle 3: Physical and mechanical characteristics of EVA.

\begin{tabular}{lc}
\hline Property & Value \\
\hline Density $\left(\mathrm{g} / \mathrm{cm}^{3}\right)$ & 0.9335 \\
Tensile strength $(\mathrm{MPa})$ & 29 \\
Melting point $\left({ }^{\circ} \mathrm{C}\right)$ & 290 \\
Flexural modulus $(\mathrm{MPa})$ & 3100 \\
Elongation at break $(\%)$ & 800 \\
Water absorption $(\%)$ & 0.07 \\
\hline
\end{tabular}

TABLE 4: The SBS natural characteristics.

\begin{tabular}{lc}
\hline Items & Properties \\
\hline Hardness $($ shore $\mathrm{A})$ & 70 \\
Density $\left(\mathrm{g} / \mathrm{cm}^{3}\right)$ & 1.03 \\
Tear resistance $(\mathrm{N} / \mathrm{mm})$ & 50 \\
Tensile strength at break $\left(\mathrm{kg}_{\mathrm{f}} / \mathrm{cm}^{2}\right)$ & 65 \\
Elongation at break $(\%)$ & 725 \\
Physical form & Pellet/crumb \\
\hline
\end{tabular}

\section{Results and Discussion}

3.1. Ductility. The experiment was performed at $25^{\circ} \mathrm{C}$ to inspect the stretchy features of the unamended as well as amended bitumen. Consequences explained in Figure 1 demonstrated that the ductility of the pure bitumen caused a huge decrease as the additive concentration increases. So, when the modifier content grows to $7 \%$, the ductility of ELTs, SBS, and EVA was decreased from $150 \mathrm{~cm}$ to $11 \mathrm{~cm}, 18 \mathrm{~cm}$, and $14 \mathrm{~cm}$ with percentage decrease of $93 \%$, $88 \%$, and $91 \%$ of the base asphalt values, respectively. Considerable decrease in ductility signalized to the high wastage in the resilience of the neat asphalt. The massive losses happened by adding ELTs, SBS, and EVA additives at a concentration ranging from $4 \%$ to $7 \%$ regarding the weight of each pure bitumen binder enhancer.

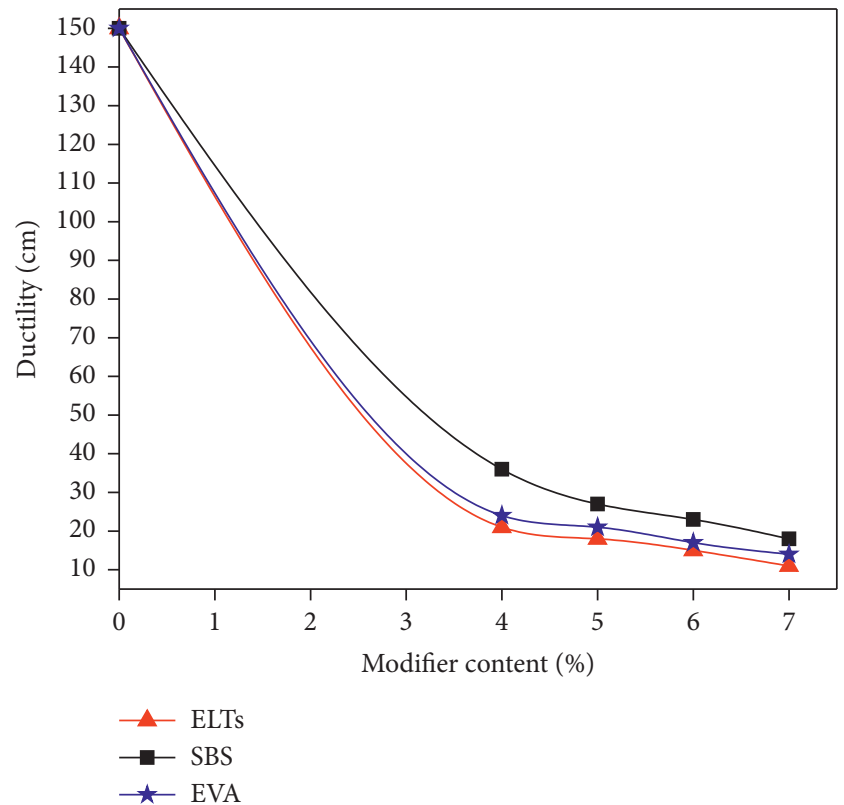

Figure 1: Modifier effect on ductility.

3.2. Kinematic Viscosity. Rotational viscosity experiment outcomes displayed that the refined asphalt viscosity boosts linearly together with the growing of ELTs, EVA, and SBS concentration as shown in Figure 2. It can be seen that the effects of modifier properties on modified asphalt represented by ELTs, EVA, and SBS-modified asphalt binders are basically different. It is observed that the viscosity gained from ELTs-amended bitumen binders was greater than theses acquired from the pure asphalt binder, EVA- and SBS-amended asphalt by $204 \%, 14 \%$, and $28 \%$, respectively, at a concentration of $7 \mathrm{wt}$. \% of each modifier. The impact of ELTs, EVA, and SBS modifiers on viscosity proved that the addition of the mentioned additives leads to a boost in viscosity. Conclusions showed that the viscosity was increased from $0.411 \mathrm{~Pa}$. $s$ to $1.249,1.0986$, and $0.9785 \mathrm{~Pa}$. $\mathrm{s}$ after the addition of 7 wt. \% of ELTs, EVA, and SBS, respectively. The high viscosity is in demand to get better rutting impedance and stress or reversal cracking resistance in the flexible pavement. In comparison among the viscosity consequences of utilized additives, ELTs have a higher viscosity than those of EVA- and SBS-amended asphalt. As a result, ELT is an extremely useful modifier for promoting the 


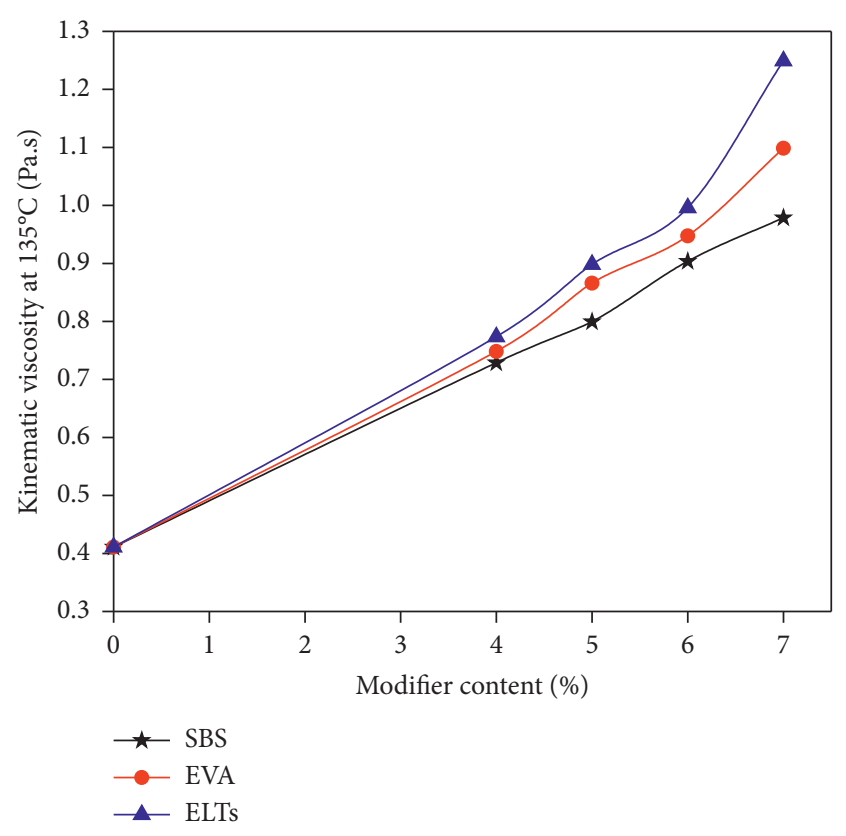

Figure 2: Modifier effect on viscosity.

rutting strength and fatigue or reversal cracking resistance in flexible pavement followed by EVA and SBS additives.

\subsection{Dynamic Shear Rheometer}

3.3.1. Rutting Parameter and Complex Shear Modulus. The obtained results of DSR experiment are presented in Figures 3 and 4 . To realize the behaviours of every enhancer clearly, the optimum concentration amount of each additive was chosen (e.g., EVA 7\%, SBS 7\%, and ELTs 7\%). Figure 3 displays that rutting parameter was reduced with temperature increase. Moreover, amounts gained from ELTsamended asphalt were more than the values from EVA- and SBS-enhanced bituminous. The decrease in $\mathrm{G}^{*} / \sin (\delta)$ incremented the right grade temperature of the bitumen material. According to rutting parameter $\left(\mathrm{G}^{*} / \sin (\delta)\right)$ amounts, ELTs-enhanced binders have a high impedance to permanent deformations than EVA and SBS counterparts.

The increase of $\mathrm{G}^{*}$ at all modified binders concerning base asphalt indicates to the grade of alteration in textures and components of asphalt to allow additives affecting mechanical features of amended asphalt. $G^{*}$ value was minimized when temperature rises, as shown in Figure 4. Outcomes confirmed that the amount of $\mathrm{G}^{*}$ was growing when the modifier content increased for each modifier concentration ranging from $4 \%$ to $7 \%$. Notice that Figures 3 and 4 were derived from the ideal proportions of the utilized additives.

3.3.2. Strain Sweep Test. The asphalt rheological characteristics were uncommonly boosted by adding 4 to $7 \%$ of ELTs, EVA, and SBS modifiers. Substantially, the hightemperature characteristics were higher in ELT-modified bitumen than those of EVA- and SBS-amended bitumen

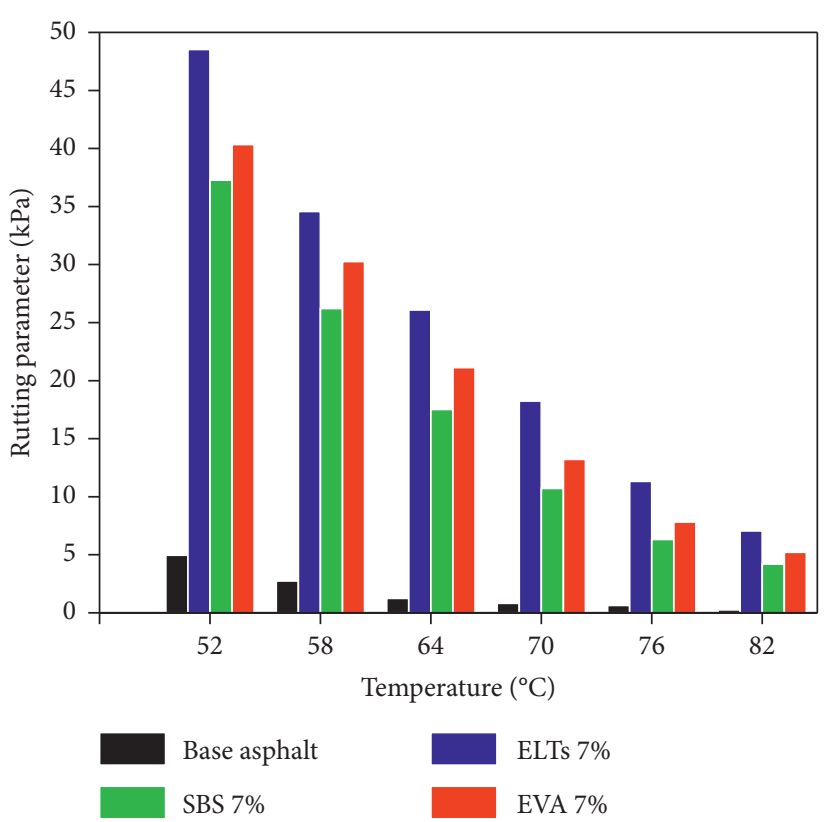

Figure 3: Rutting parameter $\left(\mathrm{G}^{*} / \sin (\delta)\right)$-temperature charts.

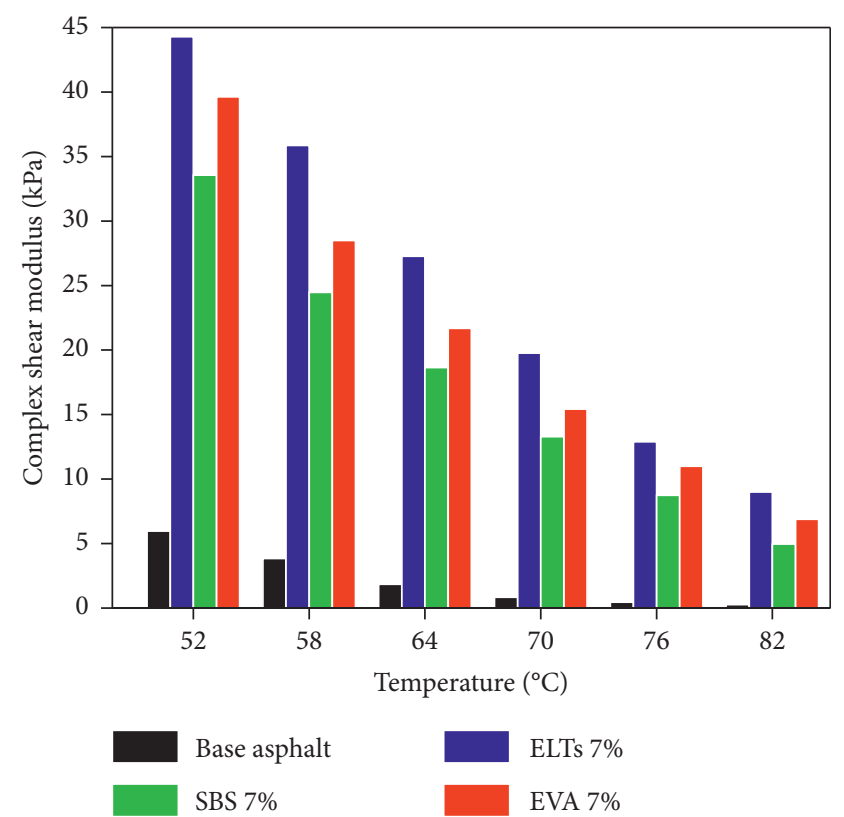

Figure 4: Complex shear modulus-temperature charts.

binders for all used concentrations. The phase angle $(\delta)$ as a function in temperature for amended and unenhanced asphalt binders in medium and high temperature is illustrated in Figure 5. It is appeared that with increasing temperature, $\delta$ increased, and $\delta$ reduced with increasing of ELTs, EVA, and SBS concentrations. Meanwhile, the reduction at phase angle values upgraded the flexible response of bitumen binder and admixture elasticity. Note that Figure 5 was plotted from the ideal concentrations of the additives. Overall, conclusions proved that the ELTs-amended binders have a lower phase angle. Therefore, ELTs are the most favourite to reinforcing asphalt at moderate- and high-temperature rendering. Also, 


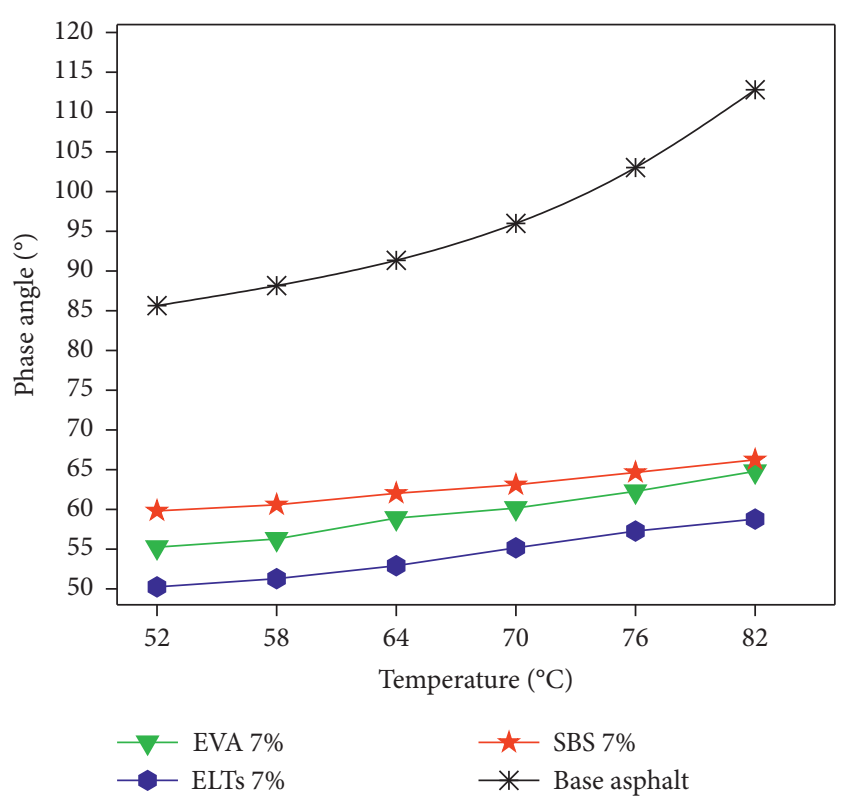

Figure 5: Phase angle $(\delta)$-temperature curves.

observed in Figure 6, at temperatures above the $70^{\circ} \mathrm{C}, 6 \%$ ELTs- and 7\% SBS-enhanced binders behave adjacent to each other and present a quite small divergence. Characteristics of 5\% ELTs and 5\% EVA amended bitumen also approached each other and exhibited tiny variation at temperatures more than $70^{\circ} \mathrm{C}$. Phase angle-temperature relationship explained that ELTs hold the more extensive linear viscoelasticity areas. Meanwhile, lowering in phase angle ameliorates the flexible response of asphalt binder and admixture resilience. Results proved that ELTs-enhanced asphalt has the highest rutting resistance. The phase anglecomplex shear modulus relationship of the utilized modified binders is clarified in Figure 7. In Figure 7, ELTs curves at ELT-modified binders have better compatibility of the modifier with virgin asphalt binder results in significantly reduced phase angles at higher temperatures matching to the other enhancers.

3.4. Epi-Fluorescence Microscopy. In the fluorescence microscopy test, the microscopic regulation is at all events equipped with a discriminatory fluorescent camera to record the images. The SBS, EVA, and ELTs implemented with content of $7 \%$ by the weight of each neat asphalt modifier. To allow modifier swelling to reach an equilibrium condition, the modified binders were allowed to cool to room temperature and stand overnight after mixing. The neat and modified asphalt samples were subjected to the rolling thin film oven (RTFOT) ageing test to confirm that there is an evolution in the microstructure.

ASTM D36 method for sample preparation for epifluorescence analysis was investigated. During microscopic analysis, the exposed surface started to flow with ultraviolet light (UV) exposure. The heat from the lens heats the sample, which in turn flows. To prevent the specimen from flowing and prevent bitumen-air interaction, two glass slides were used to sandwich the sample as in Figure 8.

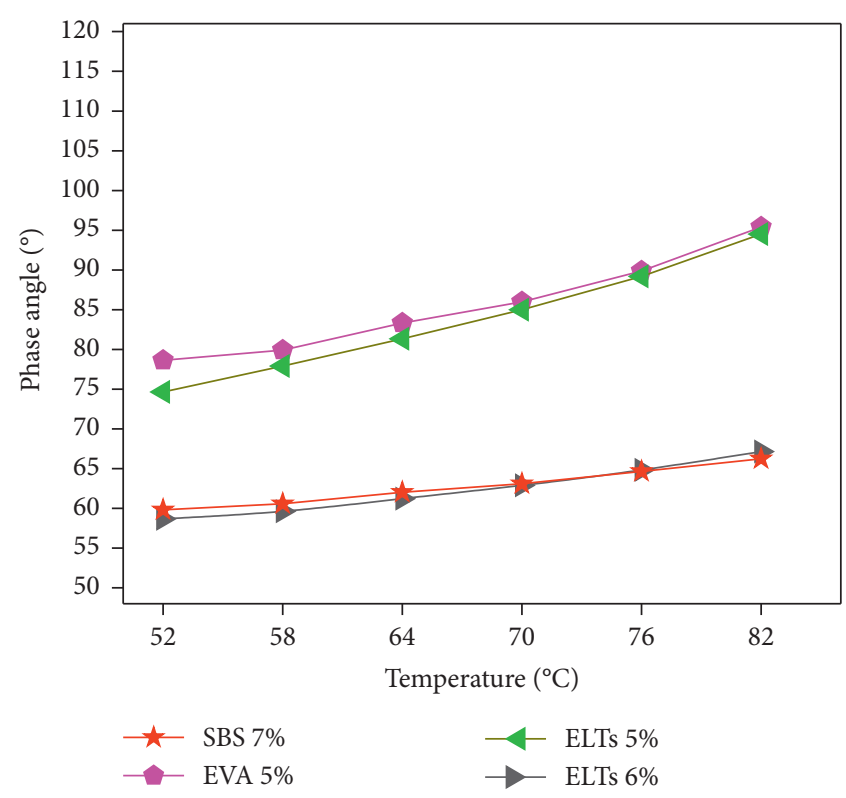

FIgURE 6: Adjacent characteristics of used modifiers.

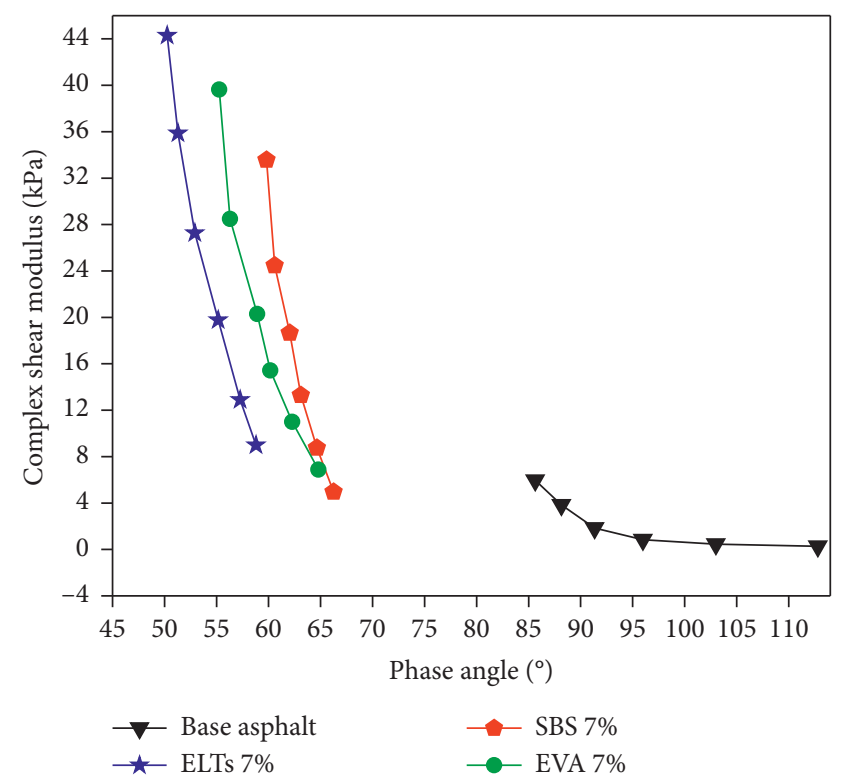

FIgURE 7: Phase angle $(\delta)-\mathrm{G}^{*}$ relationship of the modified binders.

Figure 9 shows that when the modifier content is $7 \%$ (optimum dosage), the emulsification effect of the EVA and SBS modifiers was not good, the poor compatibility (bad adsorption) and agglomeration phenomenon are obvious, and the insufficient adsorption of modifier leads to the rapid agglomeration.

Epi-fluorescence test results explained that the modifierrich phase appears as bright discrete units, as clarified in Figures 9(a)-9(d) which illustrated the modifier dispersion that observed under the microscope. Before ageing process (e.g., RTFOT), samples tend to have a good dispersion of the modifier; these samples were ELT-modified samples as seen in Figure 9(d). 


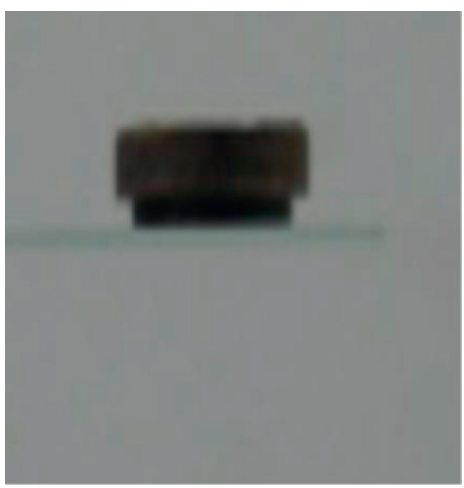

(a)

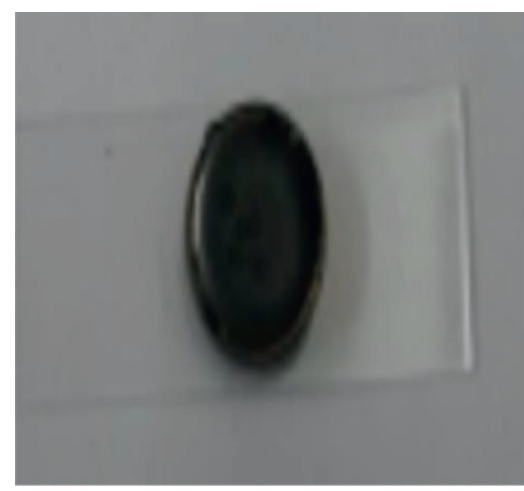

(b)

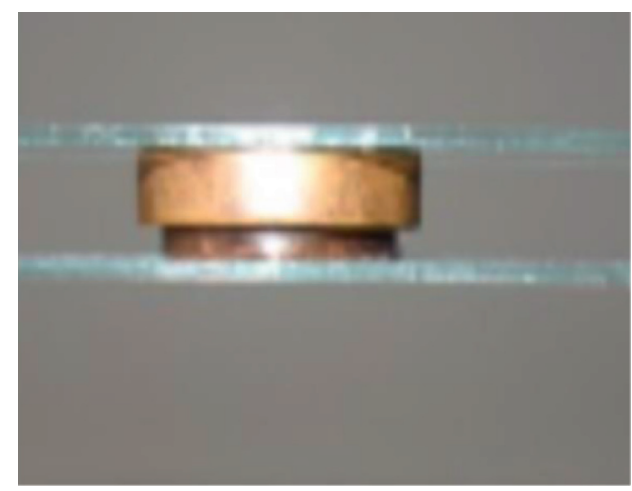

(c)

FIGURE 8: Bitumen inside a brass ring: (a) side view, (b) vertical view, and (c) brass ring with two glass slides.

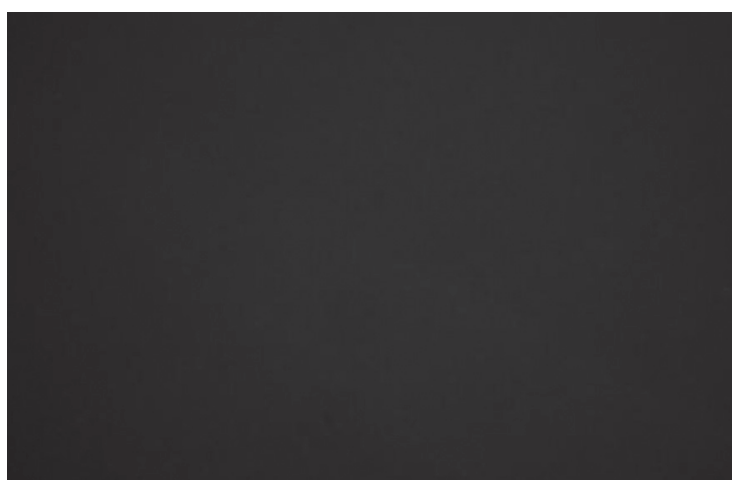

(a)

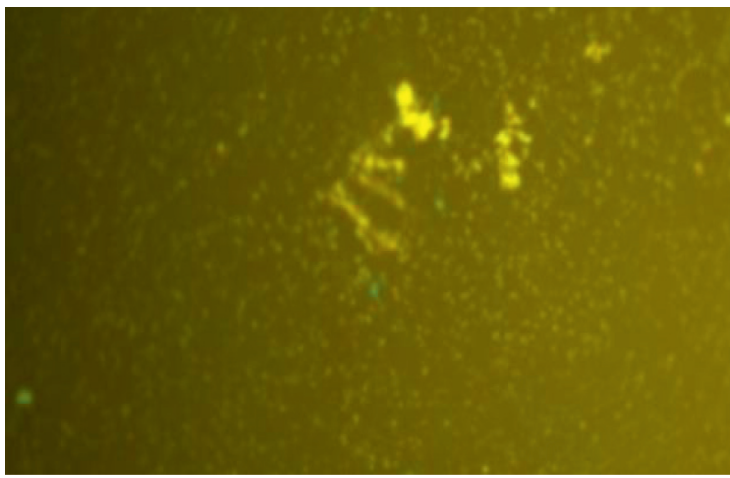

(c)

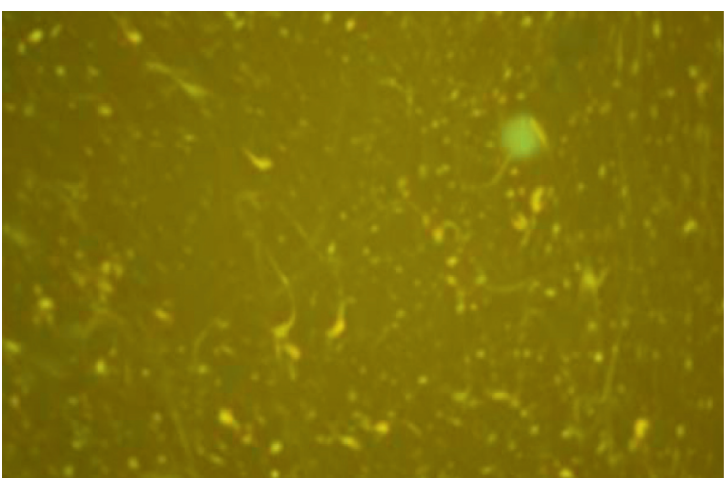

(b)

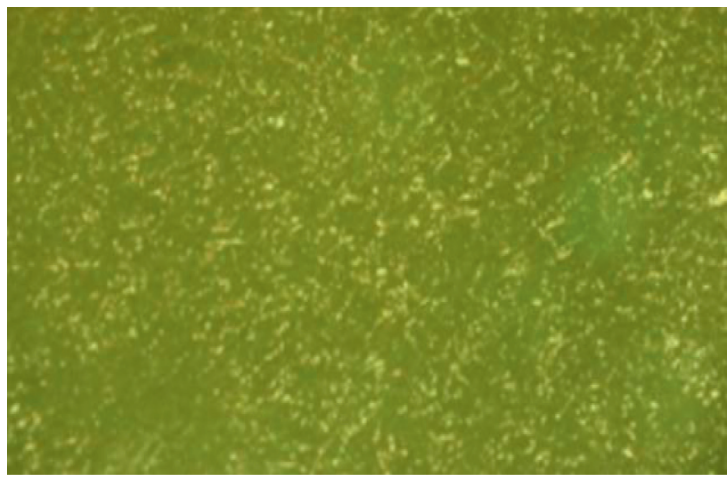

(d)

FIGURE 9: Fluorescent images of modified bitumen at $180^{\circ} \mathrm{C}$ before ageing: (a) pure asphalt, (b) SBS, (c) EVA, and (d) ELTs.

The microscopic test outcomes showed that some specimens tended to further cluster to form discrete units of a modifier, as shown in Figures 9(b) and 9(c). As agglomerates appeared from Figure 9, it is observed that some modifiers are not optimally distributed, and this is specified as poor compatibility. Figure 10 shows that the ELTs and EVA are generally in a complete dispersion, and the agglomerate is small, indicating that the ELTs and EVA modifier binders have good mechanical properties and are not easily damaged during construction. Based on analysis of the modified samples after the RTFOT process, some of the specimens tended to have a more excellent modifier dispersion as in ELTs and EVA-modified asphalt samples as seen in Figures 10(a) and 10(c).

The adhesion between modified asphalt and aggregate varied depending on the type of modified asphalt or aggregate and temperature. The epi-fluorescence microscopy observations showed that the excellent compatibility was formed at the interface between asphalt binder (EFTs) and modifier, which was believed to amend the interfacial bonding between modified asphalt binder and aggregate. 


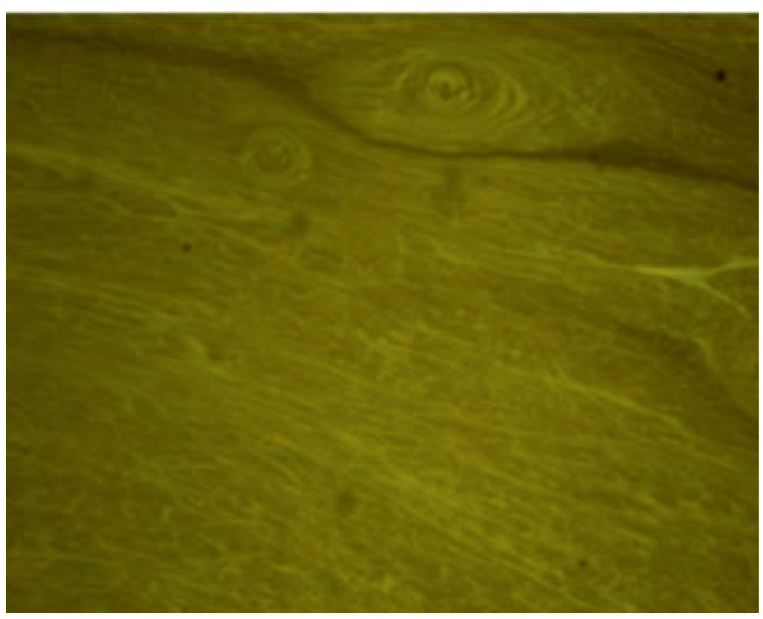

(a)

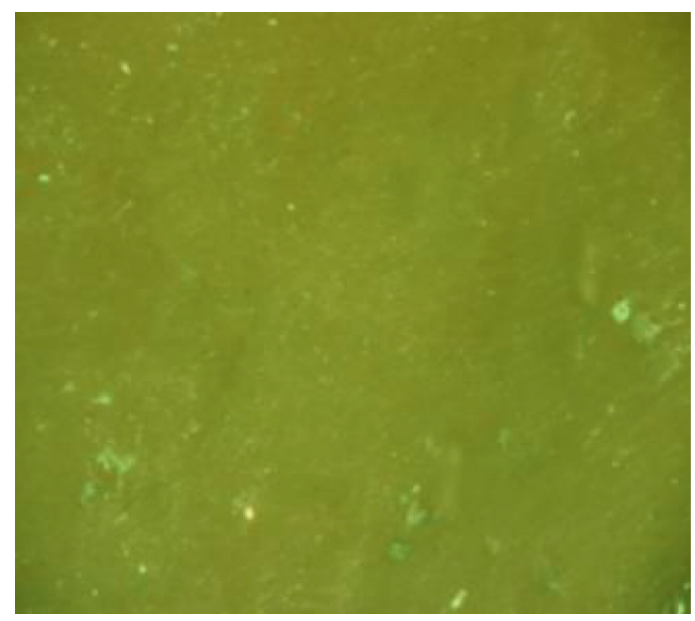

(b)

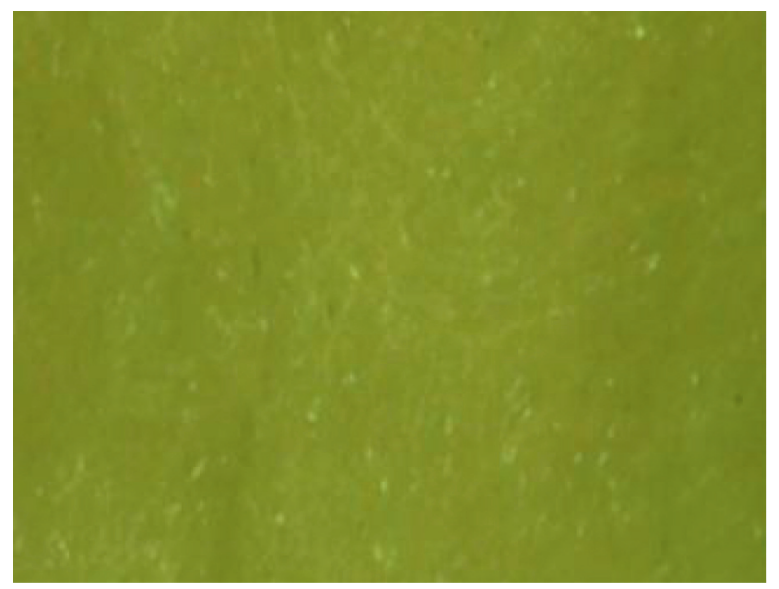

(c)

Figure 10: Fluorescent images of modified bitumen at $180^{\circ} \mathrm{C}$ after ageing: (a) EVA, (b) SBS, and (c) ELTs.

\section{Conclusions}

Depending on the consequences of the current study, the following conclusions can be drawn:

(1) The increase in viscosity with the addition of $7 \%$ ELTs was $14 \%$ and $28 \%$ more than viscosity with addendum of 7\% EVA and SBS, respectively. Hence, ELTs are considered as the most effective enhancer for their better promotion in viscosity to beneficent rutting and fatigue cracking resistance followed by EVA and SBS additives.

(2) After modification, ductility was reduced from $150 \mathrm{~cm}$ to 11,18 , and $14 \mathrm{~cm}$ by adding $7 \%$ of ELTs, SBS, and EVA, respectively. The percentage decrease in ductility was $93 \%, 88 \%$, and $91 \%$ of the base asphalt concerning ELTs, SBS, and EVA, respectively.

(3) Rutting parameter and complex shear modulus were incremented with an increase of modifier concentration and reduced with temperature increase. In contrast, the phase angle increased with temperature increase and reduced with increasing modifier concentration. Thus, values acquired from ELTs- amended asphalt were more than values gained from EVA- and SBS-adjusted bitumen. So, the ELTs modifier is the most efficient to reinforce the medium- and high-temperature rendering and permanent deformation.

(4) Epi-fluorescence microscopy demonstrated that the different morphological characteristics of the modified bitumen binders would affect their physical and rheological properties.

(5) Analysis of the enhanced bitumen samples previously and after the ageing process (RTFOT) demonstrated that ELT samples tend to have a good dispersion of the modifier. Also, ELT specimens tend to have excellent compatibility, modifier distribution, and assessing of the integrity of the modifier during production and paving of asphalt layers to improve the pavement performance.

(6) The samples tend to having larger discrete modifier agglomerates are SBS- and EVA-modified samples. Relatively, ELTs preserve their ligament with bitumen better than EVA, and SBS modifiers and ELTsenhanced asphalt displayed the lower stiffness and 
degree of ageing under the highest resistance to deformation and fatigue cracking at medium and high temperatures.

(7) ELTs were accepted as the best enhancer for their positive influence on physical and rheological characteristics, which means higher quality amended bitumen would give higher resistance to permanent deformation.

Note that this study investigated the medium- and hightemperature effects. In future studies, the authors will investigate the low-temperature properties of asphalt modified by ELTs, EVA, and SBS and the effect of these additives on the mechanical characteristics and performance of hot mix asphalt.

\section{Data Availability}

The data used to support the findings of this study are available upon request to the corresponding author.

\section{Disclosure}

Because all the experiments related to this work have been conducted in the company's laboratory which cooperated with Southeast University, this research received no external funding.

\section{Conflicts of Interest}

The authors declare that there are no conflicts of interest regarding the publication of this paper.

\section{Acknowledgments}

The authors express sincere thanks to the "Jiangsu Tiannuo Advanced Material Technology Co., Ltd., China," for patronizing this work.

\section{References}

[1] F. Khabaz and R. Khare, "Glass transition and molecular mobility in styrene-butadiene rubber modified asphalt," The Journal of Physical Chemistry B, vol. 119, no. 44, pp. 14261-14269, 2015.

[2] S. A. Elfadil, F. C. Pei, and M. L. Yi, "Effects of ethylene vinyl acetate and nanoclay additions on high-temperature performance of asphalt binders," Construction and Building Materials, vol. 169, pp. 276-282, 2018.

[3] N. Z. Habib, I. Kamaruddin, M. Napiah, and I. M. Tan, "Effect of mixing process on polypropylene modified bituminous concrete mix properties," Journal of Civil, Environmental, Structural, Construction and Architectural Engineering, vol. 5, no. 10, pp. 477-482, 2011.

[4] J. D. Lin, S. H. Chen, P. Liu, and J. N. Wang, "Modified toughness used to evaluate the effect of polymer modified asphalt on SMA," Journal of the Chinese Institute of Engineers, Chinese Institute of Engineers, vol. 27, no. 7, pp. 1013-1020, 2004.

[5] S. S. Galooyak, B. Dabir, A. E. Nazarbeygi, and A. Moeini, "Rheological properties and storage stability of bitumen/SBS/ montmorillonite composites," Construction and Building Materials, vol. 24, no. 3, pp. 300-307, 2010.
[6] R. Li, F. Xiao, S. Amirkhanian, Z. You, and J. Huang, "Developments of nano materials and technologies on asphalt materials-a review," Construction and Building Materials, vol. 143, pp. 633-648, 2017.

[7] R. Dong, M. Zhao, and N. Tang, "Characterization of crumb tire rubber lightly pyrolyzed in waste cooking oil and the properties of its modified bitumen," Construction and Building Materials, vol. 195, pp. 10-18, 2019.

[8] S. C. Bamber, R. K. Blackman, J. K. Ambrose, and C. Taylor, "Durability of asphalt mixtures: effect of aggregate type and adhesion promoters," International Journal of Adhesion and Adhesives, vol. 54, no. 5, pp. 100-111, 2014.

[9] T. W. Cao, S. P. Wu, C. H. Liu, and T. Zhang, "Shear resistance properties of TPS modified bitumen binders and asphalt mixtures," Central South University of Technology, vol. 15, no. 1, pp. 434-437, 2008.

[10] M. B. Bouraima, X.-H. Zhang, S.-W. Zhou, and Y. Qiu, "Impact of viscosity modifier on asphalt properties used for bus rapid transit lane in Chengdu," Journal of Modern Transportation, vol. 25, no. 3, pp. 185-193, 2017.

[11] S. J. Rozeveld, E. E. Shin, A. Bhurke, L. France, and L. T. Drzal, "Network morphology of straight and polymer modified asphalt cements," Microscopy Research and Technique, vol. 38, no. 5, pp. 529-543, 1997.

[12] H. Kim, Performance evaluation of SBS modified asphalt mixtures using warm mix technologies, Ph.D. Thesis, Clemson University, South Carolina, USA, 2010, https://tigerprints. clemson.edu/all_theses.

[13] K. K. Kar, S. Rana, and J. Pandey, Handbook of Polymer Nanocomposites Processing, Performance and Application, Springer, Berlin, Germany, 2015.

[14] G. Mturi, K. Mogonedi, H. Marais, and R. Ntombela, "A microstructural insight of modified bitumen using epi-fluorescence microscopy," in Proceedings of the 36th Southern African Transport, CSIR International Convention Centre, Pretoria, South Africa, July 2017, http://hdl.handle.net/2263/ 627119.

[15] A. J. Barco, G. G. Travé, F. M. Navarro, G. M. Montes, and M. C. Gámez, "Comparison of the effect of recycled crumb rubber and polymer concentration on the performance of binders for asphalt mixtures," Materiales de Construcción, vol. 66, no. 323, p. 90, 2016.

[16] H. U. Bahia and R. Davies, "Effect of crumb rubber modifiers (CRM) on performance related properties of asphalt binders," Association of Asphalt Paving Technologists, vol. 63, pp. 414-449, 1994.

[17] M. E. Zumrawi, "Effect of crumb rubber modifiers (CRM) on characteristics of asphalt binders in Sudan," Materials Science and Applications, vol. 6, no. 2, pp. 1-6, 2017.

[18] C. Bulei, M. P. Todor, T. Heput, and I. Kiss, "Directions for material recovery of used tires and their use in the production of new products intended for the industry of civil construction and pavements," in Proceedings of the International Conference on Applied Sciences (ICAS2017), vol. 294, no. 1, Hunedoara, Romania, May, 2017.

[19] T. C. Billiter, J. S. Chun, R. R. Davison, C. J. Glover, and J. A. Bullin, "Investigation of the curing variables of asphaltrubber binder," Petroleum Science and Technology, vol. 15, no. 5-6, pp. 445-469, 1997.

[20] G. Airey, "Rheological properties of styrene butadiene styrene polymer modified road bitumens*," Fuel, vol. 82, no. 14, pp. 1709-1719, 2003.

[21] Q. T. Liu, S. P. Wu, C. H. Liu, and J. G. Wang, "Investigation of rheological properties of TPS modified bitumen," Central 
South University of Technology, vol. 15, no. 1, pp. 118-121, 2008.

[22] J. R. Labarta and A. Marcilla, "Thermal treatment and degradation of cross-linked ethylene vinyl acetate-polyethylene-azodicarbonamide- $\mathrm{ZnO}$ foams. Complete kinetic modeling and analysis," Industrial \& Engineering Chemistry Research, vol. 51, no. 28, pp. 9515-9530, 2012.

[23] J. R. Labarta and A. Marcilla, "Differential scanning calorimetry analysis of the thermal treatment of ternary mixtures of ethylene vinyl acetate, polyethylene, and azodicarbonamide," Applied Polymer Science, vol. 110, no. 5, pp. 3217-3224, 2008.

[24] K. Neaylon, L. C. Van den, J. P. Wu, P. R. Herrington, D. Rogers, and P. Karan, "Performance benefits of polymer modified bitumen binders for thin surfacings," vol. 655, University of Auckland, Auckland, New Zealand, NZ Transport Agency Research Report 655, 2019.

[25] K. L. Neaylon, "Performance benefits of polymer modified bitumen binders for thin surfacings," NZ Transport Agency Research Report ID 17640287, Auckland, New Zealand, 2016.

[26] R. N. Hunter, A. Self, and J. Read, The Shell Bitumen Handbook, p. 789, 2015, http://www.icevirtuallibrary.com/ info/printbooksales, 6 th edition.

[27] B. Brule, Y. Brion, and A. Tanguy, "Paving asphalt polymer blends: relationships between composition, structure and properties (with discussion)," Asphalt Paving Technology: Association of Asphalt Paving Technologists-Proceedings of the Technical Sessions, vol. 57, pp. 41-64, 1988.

[28] H. Xiaoming and I. B. Eldouma, "Experimental study to determine the most preferred additive for improving asphalt performance using polypropylene, crumb rubber, and tafpack super in medium and high-temperature range," Applied Sciences, vol. 9, no. 8, p. 1567, 2019.

[29] J. Zhang, X. Li, G. Liu, and J. Pei, "Effects of material characteristics on asphalt and filler interaction ability," International Journal of Pavement Engineering, vol. 20, no. 8, pp. 928-937, 2019.

[30] J. Zhang, H. Tan, J. Pei, T. Qu, and W. Liu, "Evaluating crack resistance of asphalt mixture based on essential fracture energy and fracture toughness," International Journal of Geomechanics, vol. 19, no. 4, Article ID 06019005, 2019.

[31] Z. Z. Liu, X. Huang, A. Sha, H. Wang, J. Chen, and C. Li, "Improvement of asphalt-aggregate adhesion using plant ash byproduct," Journal of Materials (Basel), vol. 12, no. 4, p. 605, 2019.

[32] G. Polacco, J. Stastna, D. Biondi, and L. Zanzotto, "Relation between polymer architecture and nonlinear viscoelastic behavior of modified asphalts," Current Opinion in Colloid \& Interface Science, vol. 11, no. 4, pp. 230-245, 2006.

[33] J. V. Merighi and R. M. Fortes, "The study of permanent deformation using the static creep test," in Proceedings of the $3 \mathrm{rd}$ Eurasphalt and Eurobitume Congress, vol. 2, pp. 1711-1720, Vienna, Austria, May 2004.

[34] Z. Hossain, M. Asce, M. Zaman, M. C. Saha, and T. Hawa, "Evaluation of viscosity and rutting properties of nanoclaymodified asphalt binders," in Proceedings of the Geo-Congress 2014, pp. 3695-3702, Atlanta, GA, USA, February 2014.

[35] K. Zhong, M. Sun, and R. Chang, "Performance evaluation of high-elastic/salt-storage asphalt mixture modified with mafilon and rubber particles," Construction and Building Materials, vol. 193, pp. 153-161, 2018. 